Reporte de caso

\title{
Tercer episodio de doble contractura capsular unilateral posterior a mamoplastia de aumento
}

\section{Third episode of unilateral double capsular contracture after breast augmentation}

Ricardo Londoño-García ${ }^{1 凶}$

${ }^{1}$ Médico general, Hospital Pablo Tobón Uribe, Medellín, Colombia.

Fecha correspondencia:

Recibido: diciembre 16 de 2020.

Revisado: marzo 03 de 2021.

Aceptado: julio 03 de 2021.

Forma de citar:

Londoño-García R. Tercer

episodio de doble contractura

capsular unilateral posterior a

mamoplastia de aumento. Rev

CES Med. 2021; 35(3): 325-333.

https://dx.doi.org/10.21615/

cesmedicina.6016

Open access

C Derecho de autor

Licencia creative commons

Ética de publicaciones

Revisión por pares

Gestión por Open Journal System

DOI: 10.21615/cesmedicina.6016

ISSNe 2215-9177

ISSN 0120-8705

Publica con nosotros

\section{Resumen}

La mamoplastia de aumento consiste en insertar una prótesis dentro del tejido mamario con el fin de alterar su tamaño y forma. Actualmente, se utilizan varios tipos de implantes según su forma, tamaño y material, así como diferentes variaciones en la técnica quirúrgica. Se han descrito complicaciones asociadas por ser considerados cuerpos extraños y la más común es la contractura capsular. Se presenta el caso de una paciente de 41 años a quien se le realizó mamoplastia de aumento con implantes de silicona y de forma atípica presentó un episodio de doble contractura capsular que requirió dos intervenciones adicionales por asimetría y dolor cambiando el implante y nuevamente presentando el mismo fenómeno de manera unilateral en la mama izquierda.

Palabras clave: implantes mamarios; fibrosis; mamoplastia; reacción de cuerpo extraño.

\section{Abstract}

Augmentation mammoplasty consists of inserting a prosthesis into the breast tissue in order to alter its size and shape. Currently, several types of implants are used according to their shape, size 
and material, as well as different variations in the surgical technique. Associated complications have been described because they are considered foreign bodies and the most common is capsular contracture. We present the case of a 41 year old patient who underwent augmentation mammoplasty with silicone implants and atypically presented an episode of double capsular contracture that required two additional interventions due to asymmetry and pain by changing the implant and again presenting the same phenomenon unilaterally in the left breast.

Keywords: breast implantations; fibrosis, mammoplasty; foreign body reaction.

\section{Introducción}

El aumento mamario es uno de los procedimientos estéticos más realizados en el mundo. En 2020, en Estados Unidos se realizaron 193073 procedimientos, un número bastante significativo teniendo en cuenta la pandemia por el SARS-CoV-2; en el 2019 se registraron 287085 procedimientos, de los cuales el 89 \% fueron con implantes de silicona y 11 \% con implantes de solución salina. No se cuenta con estudios epidemiológicos en nuestro país, aunque la Sociedad Internacional de Cirugía Plástica Estética refiere que Colombia ocupa el sexto lugar en el mundo en la realización de este procedimiento quirúrgico ${ }^{(1,2)}$.

Se han descrito diferentes variaciones en la técnica quirúrgica, así como múltiples vías de acceso y planos donde alojar el implante, ya sea sub muscular, subfacial, submamario o en un plano dual, con el fin de garantizar resultados más naturales y cicatrices de menor tamaño ${ }^{(3,4)}$. La introducción en el cuerpo de materiales no biológicos puede inducir respuesta inmune y en el tejido mamario puede crearse un tejido nuevo conocido como cápsula. La contractura capsular es una complicación local que ocurre debido a una excesiva reacción fibrosa frente al implante. Las teorías indican que se desarrolla una reacción inflamatoria mediada por el factor de necrosis tumoral y citoquinas inflamatorias que llevan a una fibrosis mediante la producción de colágeno, ocasionando deformación o compresión del implante y generando senos excesivamente firmes y dolorosos. En algunos casos puede ser lo suficientemente grave y requiere una nueva intervención quirúrgica ${ }^{(5,6)}$.

En la mayoría de los casos este nuevo tejido se presenta en una sola capa y cuando se forman dos capas de tejido que se ajustan envolviendo el implante mamario se forma lo que se conoce como una doble contractura capsular, siendo la capa interna la que se encuentra en contacto con el implante y por lo general lo cubre en su totalidad, mientras que la capa externa que es la que está en contacto con el tejido mamario o el musculo ${ }^{(7-9)}$. 


\section{Presentación del caso}

Una paciente de 41 años con antecedente de hipertensión arterial, en manejo con losartán, ingresó a una institución de salud para una mamoplastia de aumento, la cual se le realizó en el año 2013. Se utilizaron prótesis de una primera marca comercial, implante sin textura, con un volumen de $350 \mathrm{cc}$ bilateral y sin complicaciones en el procedimiento o en el postoperatorio.

A los ocho meses presentó una contractura capsular del seno izquierdo clasificada como Baker Il con asimetría respecto al seno contralateral, por lo que se realizó una nueva intervención para extraer la prótesis y mejorar la asimetría. No se encontró ruptura, pero se evidenció doble cápsula finamente adherida a la prótesis, sin deformarla. Se realizó capsulotomía de ambas capas y se ajusta el bolsillo para una nueva prótesis de la misma marca, no texturizada, del mismo volumen inicial, en la mama izquierda. No se realizó intervención sobre la mama derecha.

Tres años después regresó a consulta externa por cambio en el tamaño del seno izquierdo y dolor con la actividad física. Se le ordenó una ecografía de tejidos blandos que confirmó ruptura de la prótesis, por lo que fue llevada nuevamente a intervención quirúrgica. Allí se encuentra roto el implante izquierdo, con escasa reacción inflamatoria del tejido mamario adyacente y una doble cápsula adherida a la prótesis, generando compresión y deformación, sin signos de infección. En el mismo tiempo quirúrgico se realizó también cambio del implante derecho, utilizando implantes no texturizados de otra marca comercial, diferente a la utilizada en el primer tiempo quirúrgico y por decisión de la paciente se reduce el volumen a $300 \mathrm{cc}$ de manera bilateral. El seno derecho presentaba una cápsula muy fina en comparación con el seno contralateral, sin reacción inflamatoria, se tomaron cultivos con resultado negativo. No se describieron complicaciones en el postoperatorio.

Un año más tarde asiste nuevamente a consulta por dolor en mama izquierda y nuevamente contractura capsular que en ese momento se clasifica como Baker III. La paciente decide retirarse ambos implantes y en la intervención quirúrgica se encuentra nuevamente una doble cápsula rodeando la prótesis izquierda y una significativa reacción inflamatoria. Se toma muestra del tejido mamario para estudio de patología y se cultiva el líquido inflamatorio. Se realizó una mastopexia sin implantes y se hace corrección simultánea con colgajo dermoglandular inferior. El cultivo del líquido no aíslo gérmenes aerobios y en el espécimen histológico se descartó displasia del tejido mamario. 


\section{Discusión}

El aumento de senos con implantes ha aumentado de manera significativa en los últimos 40 años y se ha asociado con varios riesgos y complicaciones, entre las que se describen metaplasia sinovial, infección crónica, seroma tardío, linfoma anaplásico de células grandes y contractura capsular ${ }^{(10-12)}$.

Los estudios revelan una incidencia de contractura capsular que oscila entre el 2,8 \% y el 20,4 \%. Una revisión sistemática reciente reporta una tasa global combinada del 3,6 \%. A pesar de la amplia gama de tasas de incidencia informadas, está ampliamente aceptado que la contractura capsular es la complicación más frecuente (tabla 1) ${ }^{(3-13)}$.

Tabla 1. Tasas de incidencia de contractura capsular después de una mamoplastia de aumento (11)

\begin{tabular}{lllll}
\hline \multicolumn{1}{c}{ Nombre del estudio } & \multicolumn{1}{c}{ Tipo de implantes } & Participantes & $\begin{array}{c}\text { Años de } \\
\text { seguimiento }\end{array}$ & $\begin{array}{c}\text { Incidencia de } \\
\text { contractura } \\
\text { capsular }\end{array}$ \\
\hline Spear et al. (9), 2014 & Natrelle, silicona redonda & 715 & 6 & 18,9 \\
\hline Blount et al. (10), 2013 & Varios & 856 & 1,2 & 2,8 \\
\hline Stutman et al. (11), 2012 & Varios & 619 & 2,4 & 7,6 \\
\hline Codner et al. (1 2), 2011 & Varios & 812 & 6 & 8,2 \\
\hline $\begin{array}{l}\text { Sevin et al. (13), 2006 } \\
\text { Implantes de silicona }\end{array}$ & texturizados (McGhan) & 210 & 8 & 8 \\
\hline $\begin{array}{l}\text { Gutowski } \text { et al. (14), } \\
1997\end{array}$ & Implantes de solución salina & 504 & 6 & 20,4 \\
\hline Total & & 3716 & $1,24-8$ & 10,6 \\
\hline
\end{tabular}

En un estudio longitudinal realizado durante 25 años, Handel et al. encuentran que la contractura capsular era la complicación más común en la mamoplastia de aumento y una relación con el tiempo: entre más tiempo se pase con los implantes mayor será el riesgo acumulado de desarrollar contractura capsular ${ }^{(13)}$.

Tradicionalmente, la contractura capsular se clasifica utilizando el sistema de clasificación de Baker, un sistema de clasificación subjetiva que se basa en los hallazgos clínicos del paciente por parte del médico (tabla 2) ${ }^{(14)}$. 
Tabla 2. Clasificación de Baker de la contractura capsular ${ }^{(14)}$

\begin{tabular}{cl}
\hline Grado & \multicolumn{1}{c}{ Características } \\
\hline I & Mama de aspecto normal, blando, de aspecto natural \\
\hline II & Mama firme, aspecto normal, se puede palpar el implante, pero no es visible. \\
\hline III & $\begin{array}{l}\text { Mama firme de apariencia anormal, puede presentar deformidad o evidenciar presencia de } \\
\text { implante. }\end{array}$ \\
\hline IV & Mama dura, en casos rígida, con dolor o sensibilidad a la palpación, anatomía distorsionada. \\
\hline
\end{tabular}

Para tratar eficazmente, o incluso prevenir, la contractura capsular, es importante comprender los mecanismos que la conducen. Se ha venido estudiado la composición celular de la cápsula y los resultados parecen sugerir un papel del sistema inmune. Los macrófagos, linfocitos y fibroblastos son las células que predominan dentro de la cápsula. Los fibroblastos se acumulan en la zona de contacto entre el implante y la cápsula y se ha visto que su número se correlaciona con el grado de contractura según la clasificación de Baker, con un mayor número encontrado en el grado IV. Puesto que los fibroblastos producen colágeno se han relacionado con la formación inicial de la cápsula. La evaluación histológica del tejido capsular ha demostrado que la mayoría del tejido está compuesto por fibras de colágeno distribuidas uniformemente ${ }^{(15,16)}$.

La orientación y organización de las fibras de colágeno parece cambiar a medida que empeora la gravedad de la contractura: las fibras se vuelven más gruesas y se establecen en forma lineal orientándose perpendicularmente a los fibroblastos para producir una forma helicoidal a medida que aumenta la gravedad del caso. Los fibroblastos, a su vez, se orientan de forma plana cuando se estudia en cápsulas que rodean implantes lisos ${ }^{(17,18)}$.

También se ha investigado la participación de los mastocitos en la patogénesis de la contractura capsular. Un estudio evidencia que los mastocitos dentro del tejido capsular expresaron renina, histamina y factor de crecimiento tumoral $\beta-1$ (TGF- $\beta)^{(19)}$. Actualmente, la investigación se centra en el papel del TGF- $\beta$. Un estudio reciente de Katzel et al. indica que este puede actuar como un interruptor maestro, lo que permite una cascada de reacciones que posteriormente conduce a la contractura capsular ${ }^{(20)}$.

Su diagnóstico es clínico y no siempre se identifican hallazgos radiológicos. En el caso descrito se documentaron tres episodios de doble capsula unilateral, se intentó cambio de marca y de volumen por voluntad de la paciente para obtener resultados estéticos, hasta que finalmente 
se decidió extraer los implantes de manera definitiva por presentar el mismo evento de manera reiterativa.

Se cuenta con cuatro hipótesis que intentan determinar la causa exacta de la formación de la doble cápsula. La primera teoría se basa en los micro y macro movimientos del implante que pueden prevenir la adhesión al tejido circundante. Se ha visto asociado con bolsillos demasiado grandes que permiten el movimiento de la prótesis ${ }^{(21)}$. En una serie de 241 procedimientos consecutivos de aumento de senos, Goes et al. advirtieron evitar la creación de un gran bolsillo donde puede haber acumulación de líquido y podría ser una fuente de infección ${ }^{(22)}$.

En segundo lugar, se mencionan las fuerzas de cizallamiento entre la cápsula original y el implante, las cuales pueden crear una separación con subsecuente neoformación de tejido capsular alrededor de la prótesis y acumulación de líquido. Hall-Findlay fue la primera en encontrar asociación de seromas tardíos con formación de doble cápsula en tres de 14 pacientes ${ }^{(23)}$.

En tercer lugar, una formación de seroma alrededor de la cápsula debido a causas infecciosas, alérgicas o hemorrágicas podrían potenciar la formación de una segunda cápsula interna. Fueron Pinchuk et al. quienes formularon esta idea durante su experiencia con 568 procedimientos de aumento de senos y en quienes se reportaron seromas tardíos en seis pacientes. Los autores enfatizan la diferencia en el fenómeno entre la aparición de un exudado fisiológico normal producido por metaplasia sinovial en oposición a un origen patológico, como linforrea, alergia, hematoma tardío, viral u hormonal ${ }^{(18,24)}$.

La cuarta hipótesis está basada en que las fuerzas de cizallamiento pueden separar el complejo entre la cápsula y el implante del tejido circundante, generando una nueva cápsula externa que produce una delaminación mecánica dentro de la cápsula ${ }^{(12,18,24)}$. Las superficies lisas de ambas capas que están en contacto con el espacio intercapsular son responsables de los micro movimientos y fricción dentro de ellas. La relevancia de esta relación dinámica reside en el aumento del riesgo de metaplasia sinovial, infección crónica, seroma tardío y posiblemente (BIA-ALCL) linfoma anaplásico de células grandes asociado a implante mamario ${ }^{(9,10,24)}$.

\section{Conclusiones}

Al ser un procedimiento quirúrgico que involucra la implantación de un cuerpo extraño, la mamoplastia de aumento o mastopexia con implantes no está exenta de complicaciones asociadas, como la contractura capsular que involucra distintas hipótesis fisiopatológicas y 
puede llegar a generar complicaciones asociadas graves como infecciones, seromas, displasia celular y alteración de los tejidos mamarios y generando reintervenciones quirúrgicas y en algunos casos cicatrices no deseadas, dismetría corporal o daño del tejido.

\section{Conflicto de intereses}

Los autores declaran no tener ningún conflicto de intereses.

\section{Bibliografía}

1. Johnson M. Breast implants: history, safety, and imaging. Radiol Technol. 2013;84(5):439M-515M; quiz 516M-520M.

2. American Society of Plastic Surgeons [Internet]. Plasticsurgery.org. [cited 2021 Jun 26]. Available from: https://www.plasticsurgery.org/news/plastic-surgery-statistics

3. Namnoum JD, Largent J, Kaplan HM, Oefelein MG, Brown MH. Primary breast augmentation clinical trial outcomes stratified by surgical incision, anatomical placement and implant device type. J Plast Reconstr Aesthetic Surg JPRAS. 2013;66(9):1165-72.

4. Nava MB, Rancati A, Angrigiani C, Catanuto G, Rocco N. How to prevent complications in breast augmentation. Gland Surg. 2017;6(2):210-7.

5. Giot J-P, Paek LS, Nizard N, El-Diwany M, Gaboury LA, Nelea M, et al. The double capsules in macro-textured breast implants. Biomaterials. 2015; 67:65-72.

6. Maxwell D, Estes M, Walcott JM, Canady JW, Hunter TD, Gache L, et al. Safety and effectiveness of silicone gel-filled breast implants in primary augmentation patients. Aesthet Surg J. 2021;41(6):NP329-35.

7. Efanov JI, Giot JP, Fernandez J, Danino MA. Breast-implant texturing associated with delamination of capsular layers: A histological analysis of the double capsule phenomenon. Ann Chir Plast Esthet. 2017;62(3):196-201.

8. Liu X, Zhou L, Pan F, Gao Y, Yuan X, Fan D. Comparison of the postoperative incidence rate of capsular contracture among different breast implants: a cumulative metaanalysis. PloS One. 2015;10(2):e0116071. 
9. Calobrace MB, Stevens WG, Capizzi PJ, Cohen R, Godinez T, Beckstrand M. Risk factor analysis for capsular contracture: A 10-year sientra study using round, smooth, and textured implants for breast augmentation. Plast Reconstr Surg. 2018;141(4S Sientra Shaped and Round Cohesive Gel Implants):20S-28S.

10. El-Haddad R, Lafarge-Claoue B, Garabedian C, Staub S. A 10-Year Prospective Study of Implant-Based Breast Augmentation and Reconstruction. Eplasty. 2018;18:e7.

11. Headon H, Kasem A, Mokbel K. Capsular Contracture after Breast Augmentation: An Update for Clinical Practice. Arch Plast Surg. 2015;42(5):532-43.

12. Nava MB, Rancati A, Angrigiani C, Catanuto G, Rocco N. How to prevent complications in breast augmentation. Gland Surg. 2017r;6(2):210-217.

13. Handel N, Cordray T, Gutierrez J, Jensen JA. A long-term study of outcomes, complications, and patient satisfaction with breast implants. Plast Reconstr Surg. 2006 Mar;117(3):757-767; discussion 768-772.

14. Maxwell GP, Brown MH, Oefelein MG, Kaplan HM, Hedén P. Clinical considerations regarding the risks and benefits of textured surface implants and double capsule. Plast Reconstr Surg. 2011;128(2):593-5.

15. Wiener TC. Comparison of Breast Augmentation Incisions and Common Complications. Aesthetic Plast Surg. 2013;37(2):475-6.

16. Spear SL, Murphy DK. Natrelle Round Silicone Breast Implants: Core Study Results at 10 Years. Plast Reconstr Surg. 2014;133(6):1354-61.

17. Tan KT, Wijeratne D, Shih B, Baildam AD, Bayat A. Tumour necrosis factor- $\alpha$ expression is associated with increased severity of periprosthetic breast capsular contracture. Eur Surg Res Eur Chir Forsch Rech Chir Eur. 2010;45(3-4):327-32.

18. Prantl L, Angele P, Schreml S, Ulrich D, Pöppl N, Eisenmann-Klein M. Determination of serum fibrosis indexes in patients with capsular contracture after augmentation with smooth silicone gel implants. Plast Reconstr Surg. 2006;118(1):224-9.

19. Steiert AE, Boyce M, Sorg H. Capsular contracture by silicone breast implants: possible causes, biocompatibility, and prophylactic strategies. Med Devices Auckl NZ. 2013;6:211-8. 
20. Katzel EB, Koltz PF, Tierney R, Williams JP, Awad HA, O'keefe RJ, et al. The impact of Smad3 loss of function on TGF- $\beta$ signaling and radiation-induced capsular contracture. Plast Reconstr Surg. 2011;127(6):2263-9.

21. Moyer KE, Ehrlich HP. Capsular contracture after breast reconstruction: collagen fiber orientation and organization. Plast Reconstr Surg. 2013;131(4):680-5.

22. Góes JCS, Landecker A. Optimizing outcomes in breast augmentation: seven years of experience with the subfascial plane. Aesthetic Plast Surg. 2003;27(3):178-84.

23. Hall-Findlay EJ. Breast implant complication review: double capsules and late seromas. Plast Reconstr Surg. 2011;127(1):56-66.

24. Pinchuk $\mathrm{V}$, Tymofii O. Seroma as a late complication after breast augmentation. Aesthetic Plast Surg. 2011;35(3):303-14. 